\title{
What Does it Mean to be a Targeted Parent? Parents' Experiences in the Context of Parental Alienation
}

\author{
Ana Tavares $\mathbb{B D}^{1} \cdot$ Carla Crespo $^{1} \cdot$ Maria Teresa Ribeiro $^{1}$
}

Accepted: 28 January 2021 / Published online: 12 February 2021

(c) The Author(s) 2021

\begin{abstract}
The term parental alienation refers to the negative influence of one parent over a child's perception of the other parent (targeted parent). The aim of this research was to gain further understanding of targeted parents' experience of parental alienation from their own perspectives. For this qualitative study, a thematic analysis of semi-structured interviews with eight participants aged between 33 and 51 years was conducted. The data from individual semi-structured interviews enabled the identification of four main themes: perception of personal impact, coping strategies, family relationships and support network. Parents identified a serious impairment of their physical and emotional well-being and mainly identified emotionfocused coping strategies to deal with this adverse ongoing situation. Furthermore, they reported changes in key relationships, with emphasis on difficulties in family and social contexts. The contribution of the findings to research and interventions with parents and families experiencing parental alienation are discussed.
\end{abstract}

Keywords Targeted parent $\cdot$ Parental alienation $\cdot$ Coping $\cdot$ Family relationships $\cdot$ Support network

\section{Highlights}

- Targeted parents report negative mental health outcomes.

- In terms of coping, targeted parents report more emotional-focused than problem-focused strategies.

- Changes in family and social support in the context of parental alienation can be detrimental to targeted parents' wellbeing.

- Multi-systemic interventions can be beneficial for improving targeted parents' well-being and family relationships.

Parental alienation is the process by which one parent negatively influences a child's perception of the other parent, identified as the targeted parent (Baker 2009; Balmer et al. 2018). While parental alienation has been the object of much discussion in the literature, there is still controversy regarding its definition and whether it ought to be conceptualized as a syndrome (Warshak 2001). However, it is consensual that alienation is a real problem (e.g., Meier 2013) and that this area of family relationships warrants more empirical research (Maturana et al. 2018). According to Bronfenbrenner's (1979) ecological model of human

Ana Tavares

ana.af.tavares@gmail.com

1 CICPSI, Faculty of Psychology, University of Lisbon, Lisbon, Portugal development, there is a mutual influence between individuals and the contexts in which they are embedded. As such, this paper acknowledges the importance of focusing on parents' individual and family experiences, as well as their perceptions of a wider support network.

Cases of parental alienation have been associated with a high-conflict separation or divorce (Baker and Verrocchio 2015; Sher 2015), however they may also occur in intact families (Baker 2006; Turkart 2002). In addition, alienation may be carried out by both mothers and fathers, although this practice has been more commonly associated with mothers since they are more frequently given custody of the children than fathers (Lowenstein 2013).

Family systems perspective has been a useful in understanding parental alienation, namely through the related concepts of triangulation and coalition in families (Baker 2009). Triangulation occurs when the child is requested by a parent to take sides against the other parent (Minuchin 
1974). This situation contributes significantly to lower levels of satisfaction with life in targeted parents (Tavares et al. 2020). In a parent-child coalition, the child tends to move into a stable coalition with one parent against the other who becomes excluded (Minuchin 1974). In parental alienation, in addition to the conflict between the parents, the child is also placed in the middle of the conflict, through insulting comments about the other parent and the fact that the child is forced to take a position (Baker and Darnall 2006). In a study with alienated parents, they identified that their children took the side of one of the parents in interparental conflicts (Baker and Darnall 2007). Thus, all family members participate and contribute to a situation of parental alienation and can determine the interaction between the child and the alienated parent given that these parent exercises less power and control over the situation (Vassiliou and Cartwright 2001).

Difficulties in co-parenting are important in order to understand parental alienation. Such difficulties may emerge in a situation of interparental conflict when the children are involved (Feinberg 2003; Moné et al. 2011). In fact, authors have pointed to how a high-conflict separation and divorce separation may intensify the occurrence of parental alienation (e.g., Gardner 2002; Vassiliou and Cartwright 2001). A set of background factors which may affect the child during a separation or divorce constitute a risk for alienation in the future. The most common risk factors for alienation consist of a history of intense marital conflict, a humiliating separation, the characteristics of the child and the personality of each parent, in addition to parenting beliefs (e.g., the alienating parent's belief that the child does not need the other parent or targeted parent's belief that the services, such as therapy or the legal system are impotent to implement changes in their relationship with the child) and behaviors (e.g., the alienating parent holding back information from the other parent regarding the child's school, health or special events and the targeted parent adopting critical behavior) (Kelly and Johnston 2001).

Previous research has identified negative consequences of parental alienation for children. In one study, greater exposure to parental alienation strategies was associated with lower children's self-esteem (e.g., Baker and Ben-Ami 2011; Baker and Brassard 2013). Additionally, through retrospective studies with adults who experienced parental alienation, exposure to parental alienation behaviors was associated with higher levels of depressive symptoms and lower quality of life (Verrocchio et al. 2019). Also, parental alienation was positively associated with difficulties in parent-child communication in adulthood, and this association was greater for participants with low self-esteem (Aloia and Strutzenberg 2018).

\section{Targeted Parents' Individual and Family Characteristics}

The literature in this field has shown that targeted parents report negative adaptation outcomes, such as depression, stress, frustration, loss, fear, helplessness and feelings of despair (Baker and Andre 2008; Baker and Darnall 2006; Vassiliou and Cartwright 2001; Poustie et al. 2018). In a recent study, the more parents perceived parental alienation tactics, the more they reported stress associated with the threat of alienation to their current and/or future wellbeing (Balmer et al. 2018). Targeted parents often experience a loss of parental role and power (Vassiliou and Cartwright 2001). They wish to maintain a relationship with their child and to seek involvement in the child's life. The difficulties encountered by targeted parents in fulfilling their parental role in addition to the concerns regarding their child's current and future mental health (Poustie et al. 2018) may contribute to the experience of a loss of control and psychological maladjustment (Balmer et al. 2018). A qualitative study showed that even when the targeted parent tries to partake with appropriate behavior, to remain neutral and not involve the child in the parental conflict, such behavior may have adverse consequences, such as this parent's increased vulnerability due to the alienating parent's hostile approach (Godbout and Parent 2012).

Targeted parents identify unfounded reasons for depreciation and children's rejection as a result of the other parent's manipulation (Baker and Damall 2007). Targeted parents specifically identify alienating behaviors as emotional manipulation, encouragement of the child's defiance and of an alliance between the alienating parent and the child, perception of being blocked from information, being the target of a denigration campaign and an overall sense of being eradicated from their child's life (Poustie et al. 2018). In fact, targeted parents perceive their relationship with their child as restricted by the other parent's behaviors, such as intercepting calls or messages when they try to make contact (Baker and Darnall 2006; Harman et al. 2016). One of the indicators of parental alienation is the decrease of contact between the targeted parent and the child, which may have a significant impact on their relationship (Vassiliou and Cartwright 2001). In parental alienation situations it is common for children to state that their thoughts are not influenced by the alienating parent, to not express guilt or remorse for having taken the side of other parent during an interparental conflict, to adopt the language of the alienating parent and to also reject the extended family of the targeted parent (Baker and Damall 2007). The children may act as secondary alienators and the alienator's extended family members also tend to follow suit and alienate (Vassiliou and Cartwright 2001). Thus, besides the impact on the targeted 
parent at an individual level, parental alienation also affects family relationships.

Nevertheless, despite feelings of despair, frustration and isolation, targeted parents appear to be resilient and seek out coping strategies (Maturana et al. 2018). Coping strategies refer to how people respond to stressful events in order to minimize the perceived threat and reduce stress. These strategies may be problem-focused (attempts to directly modify or eliminate the sources of stress) or emotionfocused (attempts to reduce or manage the emotional distress associated with the situation; Lazarus and Folkman 1984). To date, there is a scarcity of research on the coping strategies of targeted parents. One exception is the qualitative data provided by an online survey with 126 targeted parents residing in several countries (Australia, Belgium, Canada, India, Ireland, New Zealand, United Kingdom and the United States; Poustie et al. 2018). Despite the negative emotions emerging from a situation of parental alienation, the above-mentioned parents identified active coping strategies and reported having a positive attitude when dealing with parental alienation. One example of the positive attitude was hope of reunification, which appeared to be a protective factor against mental health problems (Poustie et al. 2018). In this study, parents opted to engage in therapy, to find social support and invest in self-education, acquiring knowledge as a coping strategy as they strove to raise awareness for others in the community (Poustie et al. 2018).

\section{Targeted Parents and the Wider Systems}

When a situation of parental alienation occurs, it is common for targeted parents to seek the help of mental health and legal professionals (Baker 2010). A recent systematic review found that targeted parents were dissatisfied with these types of formal support (Maturana et al. 2018). Individuals who self-identified as targeted parents reported dissatisfaction with most of their attorneys and identified suffering with anxiety and depression during the legal proceedings involving a legal episode regarding visitation, access to the child, custody decision or parenting time, and also high levels of dissatisfaction with the final decision (Baker 2010). Moreover, parents indicated dissatisfaction with health services as they perceived a lack of information in the mental health care system with regard to parental alienation cases; this promoted a sense of helplessness and hopelessness in these parents (Poustie et al. 2018). The intersection between the legal and mental health aspects of parents in a situation of parent alienation is important given that anxiety and depression are counter-productive during the legal process, impairing the perception of self-control, among other factors (Baker 2010).

\section{This Study}

Targeted parents are exposed to high levels of stress and experience psychological symptoms in a context of family adversity. However, studies addressing these parents' experiences are still surprisingly scarce (Balmer et al. 2018; Maturana et al. 2018). This research sought to address this gap by examining the experiences of eight self-identified targeted parents, based on their own perspectives, via an indepth semi-structured interview. More specifically, the meanings taken from targeted parents' individual, family and support network experiences of living a situation of parental alienation were explored.

\section{Method}

The data of this study was collected by means of semistructured interviews (Kvale 2007). Data analysis was carried out by means of a thematic analysis that organizes, describes and enables themes to be reported (Braun and Clarke 2006). The recommendations for designing and reporting qualitative research were taken into account (Levitt et al. 2017). In order to guarantee the integrity of the data, during the process of analysis particular attention was paid to the data emerging from participants' narratives and only afterwards were the theoretical frameworks used to reflect upon the results (Levitt et al. 2017). Furthermore, the RATS guidelines for qualitative research (meaning the Relevance of study question; appropriateness of qualitative method; transparency of procedure and soundness of interpretive approach) were taken into consideration, by presenting the purpose of the research, the appropriateness of the qualitative method, the procedures used to recruit and select participants, the ethical procedures and the soundness of the interpretive approach during the analysis (Clark 2003). The ethical principles of psychologists and the code of conduct of the American Psychological Association (2016) were duly considered for this study, which was approved by the Ethics Committee of the Faculty of Psychology of the University of Lisbon.

\section{Procedure}

Participants were recruited online through social media advertising a research project on parental alienation and via associations working directly with such cases. Ethics guidelines for online data collection (Barchard 2003) were taken into account and brief information on the aims of the research and the role of the participants and researchers was provided. The inclusion criteria for participation in this study were (1) being a parent with a child under the age of 18 years, (2) being a parent who identified him/herself as 
experiencing a situation where the other progenitor took physical or psychological action to promote a distance between him/her and the child, also known as alienation.

Participants completed an online survey including a sociodemographic information sheet and self-report questionnaires, a protocol designed for another quantitative study which was part of a wider research project. At the end of the protocol, parents were invited to provide their email addresses if they wished to continue participating in the second part of the study which comprised individual interviews. All the participants who had provided their email addresses were contacted by the main researcher and interviewed individually. Prior to the interview, we informed consent was obtained from all the participants to record the audio interviews. The latter were held at the Faculty of Psychology, University of Lisbon and lasted on average $1 \mathrm{~h}$ and $25 \mathrm{~min}$, ranging from between 1 and $1 \mathrm{~h}$ and $59 \mathrm{~min}$. At the end of the interview, participants were encouraged to use the main researcher's email in case they required further clarification on the study. Participants were also informed about the available counseling/therapeutic options at the Community Clinic at the Faculty.

\section{Participants}

The participants consisted of 5 males and 3 females $(N=8)$ aged between 33 and 51 years $(M=42.5 ; S D=6.91)$. Half had completed high school, one had completed middle school, and three held university degrees. All but one participant were employed at the time of the study. With regard to socioeconomic status (SES), five were from medium, two from low and one from high SES families. The majority of participants $(n=5)$ had only one child, two had two children and one had four children. The ages of the children ranged from 2 to 20 years $(M=11.92 ; S D=5.33)$. Four participants were divorced, three were single and one participant had remarried. At the time of the study, three participants were receiving mental health support, while the remaining five were not. The sociodemographic data of the sample are presented in Table 1.

\section{Measures}

A semi-structured interview was selected as the most appropriate instrument for collecting data for this study. We aimed at conducting interviews that would allow simultaneously for a structure and purpose determined by the interviewer and for an understanding of the patterns and themes emerging from the participants' own perspective when describing their experience of parental alienation (Kvale 2007). An interview script with eight thematic blocks was constructed. This research focuses on the analysis of three of these thematic blocks: Individual and family experiences (e.g., How have you been dealing with this issue in your family?; How have you been able to overcome the difficulties you have been dealing with?) Sources of support (e.g., Has anyone been helping you deal with this situation?; Who is this person and how has he/she helped you/ who are these people and how have they helped you?) and Adaptation processes (e.g., What factors have been important for you to be able to adapt to this situation?).

Additionally, parents also completed a sociodemographic form and provided information on their age, marital status, schooling and current job status. On the basis of a wellestablished classification for Portugal (Simões 1994), information on the latter two factors (schooling and current job status) enabled a calculation of the family's SES.

\section{Data Analysis}

All the interviews were fully transcribed, and the data analysis was supported by QSR NVIVO 10 software. A thematic analysis was conducted and followed the steps recommended by Braun and Clarke (2006). The analysis was carried out by the first author, who regularly discussed the reflections and analysis process with the research team. Firstly, the main researcher's familiarization with the data was prioritized by actively and repeatedly reading the transcribed interviews in search of meanings and thoughts. The next step involved generating initial codes extracted

Table 1 Participants' sociodemographic characteristics $(N=8)$

\begin{tabular}{|c|c|c|c|c|c|c|c|c|}
\hline Sex & Age & School degree & Work & SES & Marital status & Mental health support & Number of children & Children ages (years) \\
\hline Female & 51 & University & Employed & Medium & Remaride & No & 4 & $20,18,16,12$ \\
\hline Female & 48 & High school & Employed & Medium & Divorce & Yes & 1 & 6 \\
\hline Male & 38 & High school & Employed & Medium & Single & No & 1 & 8 \\
\hline Male & 33 & Middle school & Employed & Low & Single & No & 1 & 13 \\
\hline Male & 51 & High school & Unemployed & Medium & Divorce & No & 2 & 13,18 \\
\hline Male & 40 & University & Employed & High & Single & No & 1 & 7 \\
\hline Female & 43 & University & Employed & Medium & Divorce & Yes & 2 & 9,13 \\
\hline Male & 36 & High school & Employed & Low & Divorce & Yes & 1 & 2 \\
\hline
\end{tabular}


directly from the data when searching for themes. Upon emergence of potential main themes, they were constantly reviewed and subthemes were also created. During the analysis process, some themes were excluded while others emerged as a result of permanent comparison of the data. One example may be observed in the changes concerning the coping strategies theme. While coping strategies had initially been identified individually, they were later grouped into problem-focused and emotion-focused coping subthemes, an option derived from the literature on coping (Lazarus and Folkman 1984). Themes were derived from data through inductive analysis (Braun and Clarke 2006).

Throughout the analyses, the main researcher carried out memoing (written records of the process of analysis) that helped clarify the essential ideas and patterns for discussion of the data. Moreover, these records proved to be helpful in the process of ascertaining whether the researcher's perspectives in any way limited the analyses and also in the discussion of the data with the team (Levitt et al. 2017).

The next step consisted of providing labels for the identified themes and generating clear definitions for each of them. The main researcher frequently returned to the initial interviews to better understand participants' voices. Additionally, a final check was performed to examine whether the themes were coherent with the data. Inductive thematic saturation, i.e., the (non)emergence of new codes or themes (Saunders et al. 2017), was deemed to have been achieved when no more themes and subthemes emerged, and at that point the process of discussion and writing up the results according to Braun and Clarke (2006) was carried out.

\section{Results}

The data analysis of the qualitative data resulted in four main themes related to parents' experience of living a situation of parental alienation: (1) perception of personal impact; (2) coping strategies; (3) family relationships and (4) support network.

The working definitions for the themes and a selection of quotes to illustrate participants' perceptions of their experiences as targeted parents are presented below. Following the quotes, the number of sources $(N=$ number of participants) and number of references $(R)$ for each theme and subtheme are presented in brackets. A map of the studies' results is depicted in Fig. 1.

\section{Perception of Personal Impact}

Perception of personal impact $(N=8, R=152)$ encompassed the reactions experienced by targeted parents, including emotional reactions $(N=8, R=143)$ and physical reactions $(N=4, R=9)$. Examples of the eleven emotional reactions are fears underlying uncertainty "I'm really scared of the future. I'm really scared, really, really, really

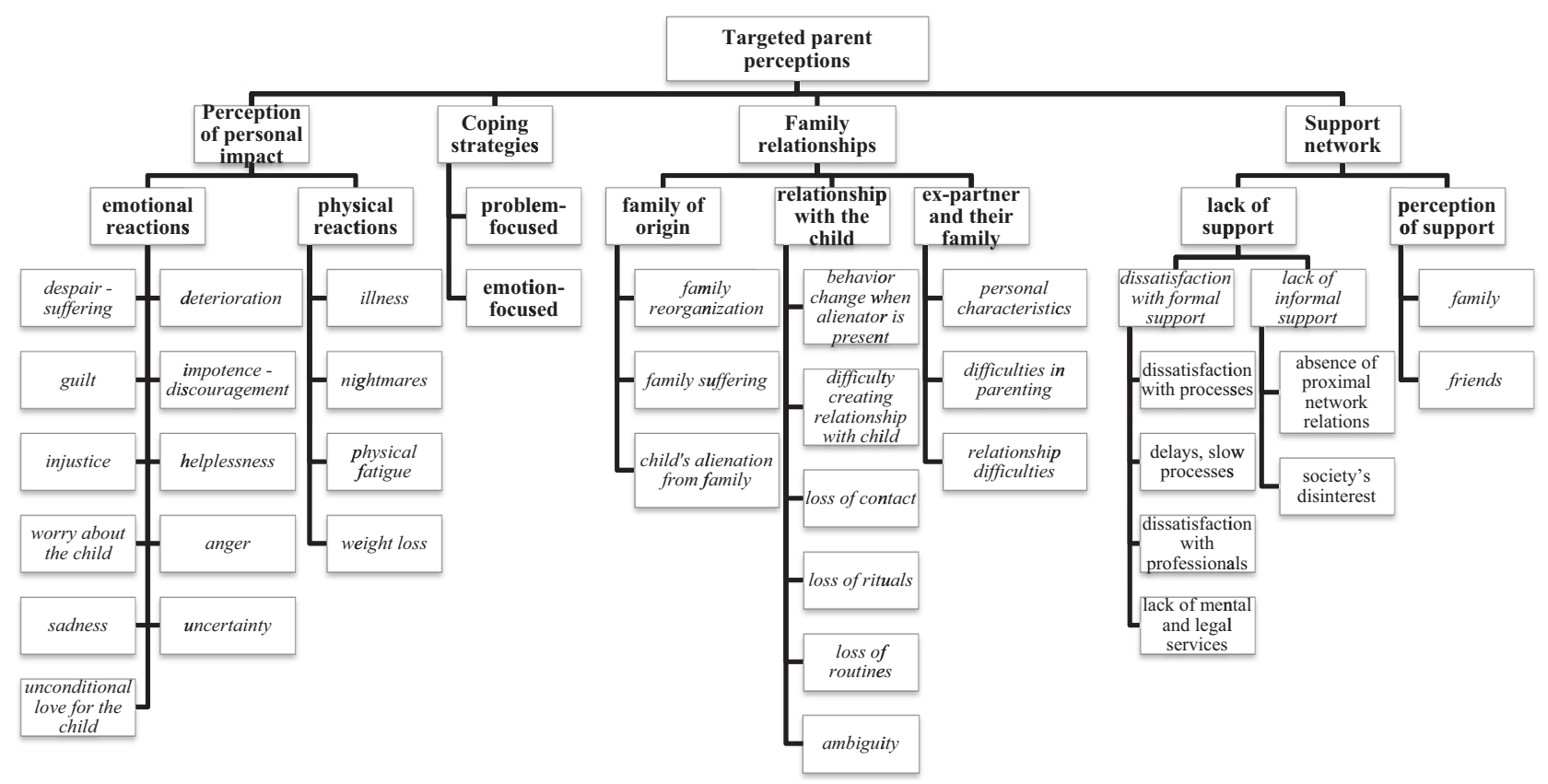

Fig. 1 Themes emerging from the data regarding the targeted parent's perception of living a situation of parental alienation. Main themes are presented in bold with a capitalized first letter; first level subthemes are written in bold with a non-capitalized first letter; second-level subthemes are labeled in italic with a non-capitalized first letter, and third-level subthemes are not presented in bold or italic and have a non-capitalized first letter 
scared of being away from my children, away from their friends, their children, scared of not being able to build any love bonds" (Claire, 51) and sadness "It's sad, I feel sad. I feel sad but I can't show it. I must look strong. (...) but I feel sad because of people's attitudes" (Richard, 33). Participants identified mainly negative emotions related to their current situation of parental alienation, experiencing negativity, guilt and injustice. However, they were still able to identify a positive emotion related to their child: unconditional love for the child, which underlined the importance of the child for parents' wellbeing.

With regard to physical reactions, we found four secondlevel subthemes, of which an illustrative example is illness "I can tell you that not long after, I was diagnosed with a chronic disease (...), all this built up stress" (Kristen, 43). In addition to the negative emotions experienced by the participants, they also felt the impact of the situation physically, reporting illnesses, physical strain, nightmares and weight loss due to the difficulties of dealing with parental alienation.

\section{Coping Strategies}

Another theme to emerge was coping strategies $(N=8$, $R=89$ ), referring to parents' strategies created in response to the stress brought by parental alienation. Parents' coping strategies were organized in two first-level subthemes, namely emotion-focused $(N=8, R=63)$, including efforts to reduce or eliminate the emotional impact of parental alienation and problem-focused $(N=7, R=26)$, including efforts to reduce or eliminate the stress event of parental alienation, as represented in Fig. 2.

Some illustrative examples of second-level subthemes of emotion-focused coping are distractions with friends, including social activities to move the focus away from the problem "some friends, when I'd say I wouldn't go, they'd go to my house and ring the doorbell and say: we're here!" (Kristen, 43) and proactivity, involving actions for dealing with parental alienation difficulties "I want to fight so that the situation is viable in the near future. I hope the other side will unbar access to our child because I wasn't born to be a part-time father" (Carl, 36). In total, the participants were able to identify nine coping strategies linked to their emotions. This may be due to the emotional burden caused by parental alienation as parents appeared to adopt strategies to minimize the emotional impact.

With regard to problem-focused coping, five secondlevel subthemes were found, such as writing journals "I have a journal where I write the most relevant things. Because in the end I want to say... this is what it is (...) the goal is to turn this into a book and at the right moment try to document this with factual evidence" (Louis, 51) and looking for information "and it's my patience, my

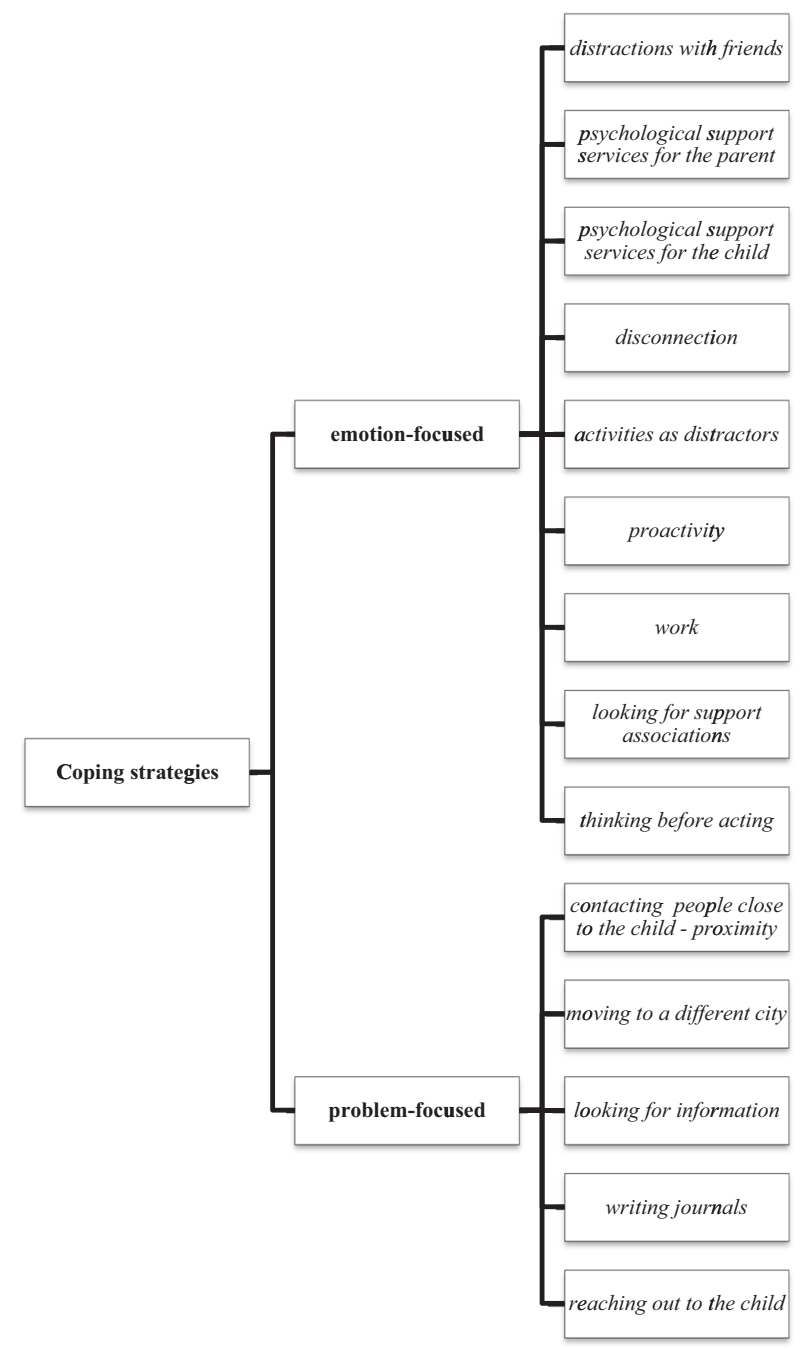

Fig. 2 Details for the theme of coping strategies: categorization within problem-focused and emotional-focused strategies

perseverance that have got me to where I am today, and also the fact that I have all the information on this subject." (Carl, 36). Participants found it more difficult to find coping strategies focusing on the problem; this may happen due to the difficulty of knowing how to act and what to do to solve their current problem. However, the strategies reported by these parents enabled them to find resources to deal with the perceived alienation and to take action to increase their personal well-being.

\section{Family Relationships}

The theme family relationships encompassed challenges experienced in relationships with family members $(N=8$, $R=339$ ). This theme included three first-level subthemes: family of origin, including relationships with the targeted parent's family of origin, such as parents and siblings ( $N=6, R=24)$; relationship with the child, referring to 
how the parent related to the child and how he/she was able/unable to participate in the child's life $(N=7, R=96)$ and ex-partner and their family $(N=8, R=219)$.

Family of origin encompassed four second-level subthemes, such as child's alienation from family "I can't even be with my child and I'm the father. Not even her grandmother, uncle, aunt or cousins can. It's total distance" (Louis, 51) and family suffering, one example being: "my mother suffers a lot because of this. She's 88 years old and she has accepted it...I know it hurts her deeply, it messes with her head because they had an amazing relationship" (Louis, 51). Parents reported difficulties in parenting as a result of the ex-partner's behaviors, as well as the child's distance from the targeted parent's family of origin as the ex-partner behaved in a way that distanced the child from the side of the family that was subject to parental alienation. This sequence of events created pain, not only to the parent, but to the entire family that had lived through a non-normative adverse situation which had brought about major changes in their web of relationships.

Within relationship with the child, five second-level subthemes were found such as loss of rituals, namely the difficulty in participating or sharing moments expected to be family-time events, such as Christmas or birthdays "There isn't a Christmas, a New Year, there aren't any birthdays, no Mother's Day, no Father's Day... I don't know what Father's Day is anymore. On Father's Day in 2014, March $19^{\text {th }}$, her mother took her phone off here." (Louis, 51) and ambiguity, in relation to who was currently part of the family. For instance, in response to the question "Do you feel like your child is present in your life?" Stephen (40) replied: "In my thoughts, yes. In my physical life, no. In thought and not much else, just in my head". Changes in the parental bond were marked by distance and loss of contact, which unsettled the relationship. Family moments like birthdays were questioned, giving rise to a sense of absence of family. This absence, coupled with the parental alienation, also fostered ambiguity due to the uncertainty of the child's role and presence in the family. Despite wishing for the child's presence in the family, the loss of contact made it difficult for the participants to refer to the child as being actually present in the family.

Finally, the subtheme ex-partner and their family included three second-level subthemes: personal characteristics, focusing on the personal characteristics of the ex-partner; difficulties in parenting, referring to challenges related to the parenting role and relationship difficulties, namely challenges related to the ex-partner or his/ her family.

Within personal characteristics of ex-partner, participants identified facts in their ex-partners' background, such as family patterns "the father left when the eldest, my ex-wife, was 12. So, she never knew what it's like to have a father and so, maybe it's not a surprise that history is repeating itself." (Louis, 51). Furthermore, criticism of the ex-partner was identified as a focus "I don't understand what is going on in her head. I think it's ignorance on her part and lack of love for the child, lack of respect for the kid and also for herself' (Richard, 33). In the participants' narrative about the ex-partner, there were high levels of criticism regarding their behavior and personality or their family patterns. The participants expressed anger and appeared to focus primarily on the ex-partner's faults.

With regard to difficulties in parenting, participants referred to difficulties in co-parenting, namely the alienating parent's attempts to push them away "Because if she meant well for her children, even if they didn't want to be with me, she'd be the first to suggest they spent time with me, and this doesn't happen, it's never happened! Ever!" (Louis, 51); "I see more alienation from the mother than I do from the child. As I usually say, we give a child a candy and they forget their problems, but it's different for the mother. Her mother is constantly bugging her" (Richard, 33). Difficulties with the ex-partner were manifested through parenting, affecting the participants' parental decisions and communication with all those involved.

Finally, within relationship difficulties (with the expartner or his/her family) participants identified formal complaints filed against them, "I was accused of mistreating them, mistreating the minor, mistreating the mother, I was accused of everything and I was innocent. And what happened to the mother? Nothing. What happened to the father? A year without seeing his child." (Braden, 38) and the difficulty in contacting the child due to the ex-partner not answering phone calls, "that week between her birthday and mine... the phones were off, the phone calls went unanswered, messenger was offline... but this had happened before." (Louis, 51). The difficulties experienced by the participants appeared to reflect their relationship with the ex-partner, who compromised not only communication, but also promoted conflicts and accusations, making the experience painful for the targeted parents.

\section{Support Network}

The support network theme included parents' perceptions of support at different contextual levels: friends and family; health and legal services and society $(N=8, R=131)$, and included two first-level subthemes lack of support $(N=7$, $R=107)$ and perception of support $(N=8, R=24)$.

In lack of support two second-level subthemes were included: dissatisfaction with formal support and lack of informal support. Dissatisfaction with formal support included four third-level subthemes: delays, slow processes "They take a long time to solve things (...) because the child is 13 and this has dragged on for 10 years and in the blink 
of an eye he's going to be an adult." (Richard, 33) and dissatisfaction with processes, referring to disagreement with the procedures for parental alienation-related cases

"and what does the police do? Sends everything to court. Until proven otherwise, the father is guilty. And what happens? The minor is taken away just in case. The father is acquitted, and what happens to the mother? Nothing. Not even a fine, not a warning, nothing. And what does she do? She files another violence complaint. And what does the court do? Just in case, they take the child away again, just in case." (Braden, 38).

Participants identified several difficulties in contacting institutions to receive formal support. This may have accentuated parents' already negative perceptions of these institutions due to the system's slowness in promoting contact with the child. These difficulties, taken together, may have led to decreasing levels of satisfaction with these services.

Some examples of the second-level subtheme lack of informal support are absence of proximal network relations "my mother and my sisters support me as much as they can, but it's not enough" (Richard, 33) and overall society's disinterest:

"We live in a society that... it's my problem so I have to be the one to deal with it, period. No one cares, no one cares. No one cares! People see it but they don't want to get involved in it, they carry on with their lives and that's it." (Louis, 51).

In addition to feeling dissatisfied with the formal support offered by institutions, participants also identified incomprehension and disinterest on the part of society, within which parental alienation was not discussed, which may explain why this situation was perceived with prejudice. Additionally, participants identified the absence of proximal network relations, which may be another area of vulnerability in terms of these parents' health and well-being.

In the subtheme perception of support, two second-level subthemes were found, namely the presence of friends "(...) has helped me get through this in a more peaceful way. My partner... and two or three friends... two" (Claire, 51) and family:

"I always have my family's support. Basically, they make sure that whenever things are more aggressive, I go and have dinner at their house, or they come to mine. My mother sometimes comes to my house midweek and sleeps there so that my child can feel the presence of family" (Phoebe, 48).
While participants felt that sufficient support was not available to them, they were still able to recognize help from specific friends and family members, which was beneficial for dealing with the emotional challenges of parental alienation.

\section{Discussion}

This qualitative research focused on the individual, family and support network experiences of eight targeted parents living a situation of parental alienation. Using semi-structured interviews, this study identified these parents' own perspectives on parental alienation, contributing to filling a major gap in the current literature (for a review, see Maturana et al. 2018). The analysis of parents' narratives enabled the identification of four main themes: perception of personal impact, coping strategies, family relations and support network.

With regard to perception of personal impact, parents who experienced parental alienation were found to identify mainly negative emotions, such as feelings of injustice or guilt, and physical consequences, such as weight loss or physical fatigue. These findings corroborate prior literature pointing to the negative implications of alienation for the targeted parent's mental health (Baker and Andre 2008; Baker and Darnall 2006; Poustie et al. 2018; Vassiliou and Cartwright 2001). The only one positive subtheme related to emotions was unconditional love for the child. However, even this positive feeling was able to accentuate the negative emotions since the targeted parents experienced difficulties in expressing their love for the child as contact was denied or hard to maintain. This is in line with the finding that severity of exposure to parental alienation tactics is positively correlated with stress appraisal for such parents (Balmer et al. 2018). Moreover, targeted parents encountered barriers to the expression of their unconditional love for the child given that the triangulation which occurs in these situations is detrimental to a significant relationship between two people in a family, due to the powerful influence of a third family member. The negative relationship between parents can also shape the child's attachment to both of them (Dallos and Vetere 2012). Targeted parents' perception of lack of control and powerlessness may affect their expression of love towards the child over time.

To date, research on targeted parents has not reported on their coping strategies within this adverse life situation. In this study, they were able to identify coping strategies to deal with this stressful situation. Using Lazarus and Folkman's framework (1984), we found that participants identified more emotional-focused than problem-focused coping strategies. Reflecting on the data, parents may have placed more emphasis on strategies to maintain an emotional 
equilibrium and regulate their distress due to the pervasive negative emotions they felt, such as injustice, sadness and despair. Furthermore, these parents appeared to have great difficulty in identifying and following an effective course of action to deal with the problem directly. This may be partially due to their perceptions of a lack of formal support on the part of institutions and society.

This study also suggests that targeted parents report significant changes in key relationships. According to the Family relationships theme, parents identified changes in family relations with different members, which compromised the relationship with the child marked by distance and loss of contact. New data emerged from the participants with regard to their relationship with the child, such as, for example, the ambiguity expressed by parents in relation to the sense of family membership. This is related to the concept of ambiguous boundaries, i.e., the lack of a definition of the role and participation of family members in a family system as a risk factor for health and well-being (Boss 2006). The difficulty of having the child present in the family's daily life and in key family events caused suffering not only to the targeted parents, but also to their families of origin.

Parents specifically worried about their lack of participation in their child's daily routines and in meaningful moments of life such as birthdays and annual celebrations. Both routines and rituals are woven in the fabric of families' lives and family rituals, and both reflect and contribute to a family's identity and cohesion (Crespo et al. 2011; Fiese 2006). The lack of these family moments (as the parent was unable to participate in the child's important moments, and likewise the child could not join the parent for his/hers) may contribute to a sense of family loss and fragmentation.

In this study, parental alienation was also found to have an influence on the Support network of targeted parents. In our data, the support of specific friends and family members contrasted with the perception of a lack of formal support from institutions and society at large. This finding is consistent with prior research, indicating that targeted parents experience dissatisfaction with formal support (Baker 2010; Maturana et al. 2018). In fact, targeted parents have to deal with different systems to try and solve a situation of parental alienation. They manifest difficulties in mental and legal support (Baker 2010) due to lack of solutions for parental alienation.

\section{Limitations and Strengths}

This study has limitations requiring consideration when interpreting the current findings. Firstly, the small size of the sample prevented us from analyzing sex differences between mothers and fathers experiencing alienation; this sample limitation also warrants caution in extending the findings to other cultural backgrounds. The participants' selection was based on the parents' own perceptions, i.e., individuals who identified who identify as living some of characteristics of parental alienation as described in our study info. Other important potentially related experiences such as intimate partner violence or previous poor relationship with their children could have been present but were not assessed in our study.

Secondly, only data from one informant (targeted parent) was collected. Although it is important to have knowledge of the specific experiences of targeted parents, from a family systems point of view, capturing the perceptions of other family members may be helpful to better understand parental alienation. Thirdly, this study only captured a snapshot in time of the individuals' experience of parental alienation. Given its complexity and frequently prolonged duration, perceptions of parental alienation are bound to change after conflictual interactions or positive moments experienced with the child that may either accentuate or lighten the difficulties experienced. Moreover, considering the qualitative research review guidelines used, namely RATS (Clark 2003), we did not have another independent member to analyze and review the data. However, the data was systematically discussed within a team of three researchers.

Despite the aforementioned limitations, the findings of this qualitative study contribute significantly to new knowledge on targeted parents whose experiences have not been sufficiently researched to date. As highlighted by Templer et al. (2016) in their systematic review, legal and psychotherapeutic support are needed when parental alienation is evident, and it is important to consider restoring relationships and resolving psychological symptoms. Our study highlights these needs, as they are supported by parents' reports. Specifically, in terms of coping strategies, this study allowed for the identification of practical strategies that targeted parents use in response to this adverse situation. Further understanding of why parents use more emotional-focused than problem-focused coping strategies could be helpful, in clinical settings, to better address parents' needs.

\section{Implications for Practice and Research}

This study's findings provide relevant clues for interventions with targeted parents who perceive their key relationships and well-being as being compromised. Understanding parental alienation using Bronfenbrenner's (1979) ecological model of human development as a reference made it possible to identify the difficulties and resources in different systems and to better provide contributions to multi-systemic interventions. 
In terms of the individual experience of the targeted parent, the literature on the coping strategies of such parents is scarce. It may be necessary to work individually on new ways of dealing with the sources of stress and help parents find other problem-solving strategies. Furthermore, our findings suggest that the participants perceived a significant negative impact on family relationships, particularly parent-child relations. This finding is in keeping with Baker and Andre's (2008) recognition that professionals ought to acknowledge targeted parents' pain and loss. The identification of individual and family fragilities, as well as the dissatisfaction with formal services may provide insights into how professionals can support targeted parents. The current findings point to the importance of health services prioritizing family relations, given that parental alienation changes the dynamics not only between targeted parents and their child but also within the extended family. Additionally, considering interventions beyond the family system appears to be especially relevant as parents may benefit from receiving more support from institutions and having their problem recognized by society at a macrosystemic level.

In terms of research on parental alienation, relevant number of significant implications have emerged from this study. Firstly, it is important to map the phenomena in time in order to understand how the parental alienation process unfolds and to identify the different stages experienced by the different family members. It is also relevant to clearly identify risk and protective factors for the emergence of parental alienation, as well as its consequences in terms of health and well-being for parents and children in the short and long term. Secondly, given the systemic and complex nature of this phenomenon that involves the whole family, it is paramount that future research includes other family members besides the targeted parents, in addition to professionals who deal with situations of parental alienation. Indeed, the role of the different family members needs to be clarified; as previously identified by Vassiliou and Cartwright (2001), extended family members in a situation of parental alienation may play a role in regulating the closeness between the child and the alienating parent, however, their participation in the alienation remains unclear and warrants more empirical investigation.

Author Contributions Conceptualized the study, collected and analyzed the data and was in charge of writing up the manuscript [Ana Tavares]; Supervised the study's conceptualization and the data analysis and revised the manuscript [Carla Crespo, Luana Ferreira and Maria Teresa Ribeiro].

Funding This work was supported by the Faculty of Psychology and the University of Lisbon.

\section{Compliance with Ethical Standards}

Conflict of Interest The authors declare that they have no conflict of interest.

Ethical Approval All procedures performed in studies involving human participants were in accordance with the ethical standards of the Faculty of Psychology of Lisbon University and with the 1964 Helsinki declaration and its later amendments or comparable ethical standards.

Informed Consent Informed consent was obtained from all individual participants included in the study.

Publisher's note Springer Nature remains neutral with regard to jurisdictional claims in published maps and institutional affiliations.

Open Access This article is licensed under a Creative Commons Attribution 4.0 International License, which permits use, sharing, adaptation, distribution and reproduction in any medium or format, as long as you give appropriate credit to the original author(s) and the source, provide a link to the Creative Commons license, and indicate if changes were made. The images or other third party material in this article are included in the article's Creative Commons license, unless indicated otherwise in a credit line to the material. If material is not included in the article's Creative Commons license and your intended use is not permitted by statutory regulation or exceeds the permitted use, you will need to obtain permission directly from the copyright holder. To view a copy of this license, visit http://creativecommons. org/licenses/by/4.0/.

\section{References}

Aloia, L. S., \& Strutzenberg, C. (2018). Parent-child communication apprehension: The role of parental alienation and self-esteem. Communication Reports, 32(1), 1-14. https://doi.org/10.1080/ 08934215.2018.1514641.

American Psychological Association. (2016). Revision of ethical standard 3.04 of the "ethical principles of psychologists and code of conduct" (2002, as amended 2010). American Psychologist, 71 (9), 900-900. https://doi.org/10.1037/amp0000102.

Baker, A. J. L. (2006). Patterns of parental alienation syndrome: a qualitative study of adults who were alienated from a parent as a child. American Journal of Family Therapy, 34(1), 63-78. https:// doi.org/10.1080/01926180500301444.

Baker, A. J. L. (2009). Adult recall of parental alienation in a community sample: prevalence and associations with psychological maltreatment. Journal of Divorce \& Remarriage, 51(1), 16-35. https://doi.org/10.1080/10502550903423206.

Baker, A. J. L. (2010). Even when you win you lose: targeted parents' perceptions of their attorneys. American Journal of Family Therapy, 38(4), 292-309. https://doi.org/10.1080/01926187. 2010.493429.

Baker, A. J. L., \& Andre, K. (2008). Working with alienated children and their targeted parents: suggestions for sound practices for mental health professionals. Annals of the American Psychotherapy Association, 11, 10.

Baker, A. J. L., \& Ben-Ami, N. (2011). To turn a child against a parent is to turn a child against himself: The direct and indirect effects of exposure to parental alienation strategies on self-esteem and wellbeing. Journal of Divorce \& Remarriage, 52(7), 472-489. https:// doi.org/10.1080/10502556.2011.609424. 
Baker, A. J. L., \& Brassard, M. R. (2013). Adolescents caught in parental loyalty conflicts. Journal of Divorce \& Remarriage, 54 (5), 393-413. https://doi.org/10.1080/10502556.2013.800398.

Baker, A. J. L., \& Darnall, D. (2006). Behaviors and strategies employed in parental alienation. Journal of Divorce \& Remarriage, 45(1-2), 97-124. https://doi.org/10.1300/ J087v45n01_06.

Baker, A. J. L., \& Damall, D. C. (2007). A construct study of the eight symptoms of severe parental alienation syndrome. Journal of Divorce \& Remarriage, 47(1-2), 55-75. https://doi.org/10.1300/ J087v47n01_04.

Baker, A. J. L., \& Verrocchio, M. C. (2015). Parental bonding and parental alienation as correlates of psychological maltreatment in adults in intact and non-intact families. Journal of Child and Family Studies, 24(10), 3047-3057. https://doi.org/10.1007/ s10826-014-0108-0.

Balmer, S., Matthewson, M., \& Haines, J. (2018). Parental alienation: targeted parent perspective. Australian Journal of Psychology, 70 (1), 91-99. https://doi.org/10.1111/ajpy.12159.

Barchard, K. A. (2003). Ethics in on-line data collection. Presentation at the Western Psychological Association Annual Convention, May, 2, Vancouver, BC.

Boss, P. (2006). Loss, trauma, and resilience: therapeutic work with ambiguous loss. United States of American: W. W. Norton \& Company.

Braun, V., \& Clarke, V. (2006). Using thematic analysis in psychology. Qualitative Research in Psychology, 3(2), 77-101. https:// doi.org/10.1191/1478088706qp063oa.

Bronfenbrenner, U. (1979). The ecology of human development. Cambridge: Havard University Press.

Clark, J. P. (2003). How to peer review a qualitative manuscript. In F. Godlee \& T. Jefferson (Eds.), Peer review in health sciences. 2nd ed. (pp. 219-235). London, UK: BMJ Books.

Crespo, C., Kielpikowski, M., Pryor, J., \& Jose, P. E. (2011). Family rituals in New Zealand families: links to family cohesion and adolescent's well-being. Journal of Family Psychology, 25(2), 184-193. https://doi.org/10.1037/a0023113.

Dallos, R., \& Vetere, A. (2012). Systems theory, family attachments and processes of triangulation: does the concept of triangulation offer a useful bridge? Journal of Family Therapy, 34(2), 117-137. https://doi.org/10.1111/j.1467-6427.2011.00554.x.

Feinberg, M. E. (2003). The internal structure and ecological context of coparenting: a framework for research and intervention. Parenting: Science and Practice, 3(2), 95-131. https://doi.org/10. 1207/S15327922PAR0302_01.

Fiese, B. H. (2006). Family routines and rituals. New Haven, CT: Yale University Press.

Gardner, R. A. (2002). Parental alienation syndrome vs. parental alienation: which diagnosis should evaluators use in childcustody disputes? The American Journal of Family Therapy, 30 (2), 93-115. https://doi.org/10.1080/019261802753573821.

Godbout, E., \& Parent, C. (2012). The life paths and lived experiences of adults who have experienced parental alienation: a retrospective study. Journal of Divorce \& Remarriage, 53(1), 34-54. https://doi.org/10.1080/10502556.2012.635967.

Harman, J., Leder-Elder, S., \& Biringen, Z. (2016). Prevalence of parental alienation drawn from a representative poll. Children and Youth Services Review, 66, 62-66.

Kelly, J. B., \& Johnston, J. R. (2001). The alienated child: a reformulation of parental alienation syndrome. Family Court Review, 39(3), 249-266. https://doi.org/10.1111/j.174-1617. 2001.tb00609.x.

Kvale, S. (2007). Doing interviews. London: SAGE Publications, Ltd. https://doi.org/10.4135/9781849208963.
Lazarus, R. S., \& Folkman, S. (1984). Stress, appraisal, and coping. New York, NY: Springer.

Levitt, H. M., Motulsky, S. L., Wertz, F. J., Morrow, S. L., \& Ponterotto, J. G. (2017). Recommendations for designing and reviewing qualitative research in psychology: promoting methodological integrity. Qualitative Psychology, 4(1), 2-22. https:// doi.org/10.1037/qup0000082.

Lowenstein, L. F. (2013). Is the concept of parental alienation a meaningful one? Journal of Divorce \& Remarriage, 54(8), 658-667. https://doi.org/10.1080/10502556.2013.810980.

Maturana, S. L., Matthewson, M., Dwan, C., \& Norris, K. (2018). Characteristics and experiences of targeted parents of parental alienation from their own perspective: a systematic literature review. Australian Journal of Psychology (1), 1-9. https://doi. org/10.1111/ajpy.12226

Meier, J. S 2013). Parental alienation syndrome and parental alienation: Research reviews. Harrisburg, PA: VAWnet, a project of the National Resource Center on Domestic Violence/Pennsylvania Coalition Against Domestic Violence.

Minuchin, S. (1974). Families and family therapy. Boston: Harvard University. https://doi.org/10.4324/9780203111673.

Moné, J. G., MacPhee, D., Anderson, S. K., \& Banning, J. H. (2011). Family members' narratives of divorce and interparental conflict: implications for parental alienation. Journal of Divorce \& Remarriage, 52(8), 642-667. https://doi.org/10.1080/10502556. 2011.619940.

Poustie, C., Matthewson, M., \& Balmer, S. (2018). The forgotten parent: the targeted parent perspective of parental alienation. Journal of Family Issues, 39(12), 3298-3323. https://doi.org/10. 1177/0192513x18777867.

Saunders, B., Sim, J., Kingstone, T., Baker, S., Waterfield, J., Bartlam, B., \& Jinks, C. (2017). Saturation in qualitative research: exploring its conceptualization and operationalization. Quality \& Quantity, 52(4), 1893-1907. https://doi.org/10.1007/ s11135-017-0574-8.

Sher, L. (2015). Parental alienation: The impact on men's mental health. International Journal of Adolescent Medicine and Health, 29(3). https://doi.org/10.1515/ijamh-2015-0083.

Simões, M. (1994). Investigações no âmbito da aferição nacional do teste das matrizes progressivas coloridas de Raven [Raven's progressive matrices: Portuguese aferition studies]. Coimbra: Faculty of Psychology and Educational Science, University of Coimbra.

Tavares, A., Crespo, C., \& Ribeiro, M. T. (2020). Psychological adaptation and beliefs in targeted parents: a study in the context of parental alienation. Journal of Child Family Studies, 29, 2281-2289. https://doi.org/10.1007/s10826-020-01742-0.

Templer, K., Matthewson, M., Haines, J., \& Cox, G. (2016). Recommendations for best practice in response to parental alienation: findings from a systematic review. Journal of Family Therapy, 39 (1), 103-122. https://doi.org/10.1111/1467-6427.12137.

Turkart, I. (2002). Parental alienation syndrome: a review of critical issues. Parental Alienation Syndrome, 18(1), 131-176.

Vassiliou, D., \& Cartwright, G. F. (2001). The lost parents' perspective on parental alienation syndrome. The American Journal of Family Therapy, 29(3), 181-191. https://doi.org/10.1080/ 019261801750424307.

Verrocchio, M. C., Marchetti, D., Carrozzino, D., Compare, A., \& Fulcheri, M. (2019). Depression and quality of life in adults perceiving exposure to parental alienation behaviors. Health and Quality of Life Outcomes, 17(1), 1-9. https://doi.org/10.1186/ s12955-019-1080-6.

Warshak, R. A. (2001). Current controversies regarding parental alienation syndrome. American Journal of Forensic Psychology, 19(3), 29-59. 